\title{
Computational-time reduction of Fourier-Based Analytical Models
}

\author{
Bert Hannon, Peter Sergeant, Member, IEEE, Luc Dupré, Member, IEEE
}

\begin{abstract}
An increasing interest in both efficient electric machines and more extensive control strategies demands evermore faster simulation tools. In that context, Fourier-based models, which combine low computational times with a high accuracy, have already proven their value. However, even Fourier-based models may encounter problems related to CPU usage. To cope with these problems the authors present a number of simple techniques to reduce the computational effort of Fourier-based models for synchronous machines. The techniques are based on simplifying the studied geometry and a qualitative analysis of the machine's time- and spatial-harmonic content. The proposed techniques are validated and a benchmark test has shown that a great reduction in computational burden can be achieved without significant loss of accuracy. The preliminary harmonic analysis gives, by far, the largest reduction of the computational burden.
\end{abstract}

Index Terms-Electric machines, analytical models, harmonic analysis

\section{INTRODUCTION}

Although the importance of traditional electric machines, such as grid-connected induction machines, is still very large, the demand for higher efficiencies is pushing electrical engineers towards new machine topologies and more complicated control strategies. The development and implementation of such machines requires a good understanding of their physics and operation. Moreover, the design process of new machines requires a fast and accurate modeling tool, suited for optimization purposes. In that light, the class of Fourier-Based (FB) models has recently gained a lot of attention [1]-[6]. The reason for its popularity is that Fourier-based modeling is extremely suited for both the study and the design of electric machines. Indeed, despite their analytical character, which enables low computational times, Fourier-based models are capable of accurately taking into account complex physical phenomena such as eddy-current reaction field [4], [5] and the slotting effect [5], [6]. Moreover, Fourier-based models also give a good insight in the machine's physics.

As already mentioned, one of the major advantages of Fourierbased models is that they can be very fast. However, if a demand for high accuracy is combined with complex machine topologies, even the computational time of traditional Fourier-based models may become unacceptably high. This is especially true when the model is used for optimization procedures with a large initial design space.

B. Hannon, P. Sergeant and L. Dupré are with the Electrical Energy Laboratory, Ghent University, Ghent, Belgium as well as with Flanders Make, the strategic research centre for the manufacturing industry, Belgium (e-mail: Bert.Hannon@UGent.be)
Although some authors of FB models have reported on numerical issues [3], [7], [8], to date no extensive study of the computational time of such models has been presented. The only available literature that discusses computationaltime reduction was presented by Ramakrishnan et al. in [9]. However, that discussion is limited to a specific class of Fourier-based models that uses Schwarz-Christoffel mapping. The goal of this paper is therefore to meet the need for more computational-efficient Fourier-based models. Moreover, those lower computational times are to be obtained with simple and very generally applicable techniques that do not compromise the model's accuracy. Because of their relative importance in the segment of innovative electric machines, the focus in this work is on Permanent-Magnet Synchronous Machines (PMSMs).

Two techniques to reduce the FB model's computational effort are considered in this work; simplifying the geometry and applying a qualitative analysis of the machine's time- and spatial-harmonic content. That analysis was performed by the authors in [10]. The results are validated using a FiniteElement Model (FEM). A benchmarking test is performed to compare the computational time of the standard Fourier-based model with the computationally efficient models.

\section{A. Assumptions}

Before discussing the work, some general assumptions have to be made. As already mentioned, this paper focuses on PMSMs. However, such machines come in a large variety. Although the work applies to a great majority of Fourier-based models for synchronous machines, some restrictions have to be adopted.

A first assumption is that the end effects are neglected. This assumption is common for analytical models and allows for a 2D approximation.

Secondly, the applied current system is assumed to be a balanced system with an odd number of phases.

Thirdly, the stator is wound using the Star Of Slots (SOS) technique. This assumption allows for the vast majority of winding topologies, ranging from simple single-layer distributed windings to multi-phase fractional-slot concentrated windings with any number of layers.

Fourthly, as in most Fourier-based models, all materials are assumed homogeneous. This implies that local saturation is not taken into account.

Finally, the rotor is assumed to be rotating at synchronous speed. This implies steady-state operation.

The above assumptions are listed as: 
- No end-effects

- Balanced system with an odd number of phases

- Winding distribution according to SOS

- Homogeneous materials

- Synchronous operation

\section{B. Paper outline}

The work is structured in three parts. Sections II and III provide the necessary background information on Fourierbased modeling and the time- and spatial-harmonic content of synchronous electric machines. Next, Sections IV and V introduce the techniques to reduce the computational effort of FB models. These sections respectively focus on simplifying the geometry and applying a qualitative analysis of the machine's harmonic content. Finally, the proposed techniques are evaluated in Section VI and the work is concluded in Section VII.

\section{FOURIER-BASED ANALYTICAL MODELS}

The goal of this section is to introduce Fourier-based modeling. Obviously, the magnetic field is not only dependent on space but on time as well. When building a Fourier-based model, the time-dependency can be accounted for directly or through time-stepping. Both techniques will briefly be discussed in the following.

\section{A. Fourier representation of the magnetic field}

Figure 1 shows a PMSM with a Shielding Cylinder (SC). The latter is a conductive sleeve that is wrapped around the magnets [4], [5]. It is often used to reduce the rotor losses at high-speed operation, but it can also be used to retain the magnets. Although the presented work applies to a broader range of PMSMs, as explained in Section I, the machine in Figure 1 will be used as an example in this section.

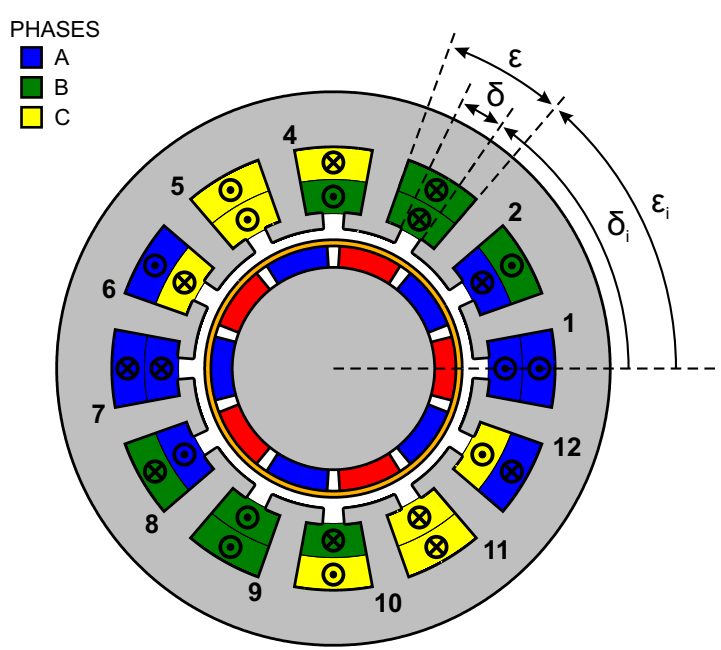

Fig. 1: Geometry of a machine with 12 slots and 5 pole pairs

The magnetic field in an electric machine has both a time $(t)$ and a spatial dependency.

It can easily be seen from Figure 1 that the shape of the example machine lends itself to using a cylindrical coordinate system $(r, \phi, z)$ to spatially describe the magnetic field. As discussed in Section I-A, neglection of the end effects implies that the magnetic field is independent from the $z$-coordinate. The time-dependency can either be taken into account directly or through time-stepping. Direct time-dependency implies using a time-dependent expression for the magnetic field. As this allows to account for eddy-current reaction field, it is the most generally valid technique [4], [5], [11]-[13], but it also implies a larger complexity. Applying time-stepping, i.e. performing multiple time-independent calculations with updated values of the rotor position, current value, etc., results in a simpler model with a lower computational burden [1] [3], [6]. However, as directly taking into account the timedependency is the most general method, it will be used in the rest of this work. Assuming synchronous rotation, the results apply to models that use time-stepping if the time-harmonic order $(n)$ is assumed to be 1 .

The magnetic field is mostly expressed using auxiliary quantities such as the magnetic scalar potential $(\psi)[1]$, [2] or the magnetic vector potential (A) [3]-[6]. As already mentioned, the magnetic field, and thereby $\psi$ and $\mathbf{A}$, are independent from $z$ if the end effects are neglected. Moreover, neglecting the end effects also implies that $\mathbf{A}$ only has a $z$ component:

$$
\mathbf{A}=A_{z} \cdot \mathbf{e}_{\mathbf{z}}=A \cdot \mathbf{e}_{\mathbf{z}}
$$

Which of both auxiliary quantities is considered has no effect on the following discussions. In this work, the more generally valid magnetic vector potential was chosen, but a completely analogue reasoning can be made for the scalar potential.

Evidently, the machine has a periodicity over both time and space. The time periodicity is the time the rotor needs to perform one revolution $\left(T_{m}\right)$, the mechanical periodicity is $2 \pi$ mechanical radians. Those periodicities can be used to express the magnetic field as a Fourier series over time and space:

$$
A(r, \phi, t)=\sum_{n=-\infty}^{\infty} \sum_{k=-\infty}^{\infty} A_{n, k}(r) e^{j\left(k \phi-n \omega_{m} t\right)}
$$

In (2), $n$ is the time-harmonic order and $k$ is the spatialharmonic order. The machine's mechanical rotational speed is denoted as $\omega_{m}$.

$$
\omega_{m}=\frac{2 \pi}{T_{m}}
$$

Because of the fact that the magnetic field has both a time and a spatial dependency, every Fourier coefficient $\left(A_{n, k}(r)\right)$ depends on both the time-harmonic order $n$ and the spatialharmonic order $k$. This means that the time- and spatialharmonic orders should not be regarded separately but as a combination. Such a time- and spatial-harmonic combination is referred to as $(n, k)$.

\section{B. Fourier-based modeling}

The fact that the magnetic field can be written as a Fourier series has been used by a great number of authors to construct analytical models of PMSMs [1]-[6]. In these Fourier-based models, Maxwell's equations and the constitutive relations are 
used to formulate a differential equation for the magnetic scalar potential or the magnetic vector potential. For the magnetic vector potential this differential equation is written as:

$$
\nabla^{2} \mathbf{A}-\mu \sigma \frac{\partial \mathbf{A}}{\partial t}+\mu \sigma(\mathbf{v} \times(\nabla \times \mathbf{A}))=\mu \mathbf{J}_{e x t}-\nabla \times \mathbf{B}_{0}
$$

where $\mu$ is the magnetic permeability, $\sigma$ is the electric conductivity, $\mathbf{v}$ is the speed, $\mathbf{J}_{\text {ext }}$ is the externally imposed current density and $\mathbf{B}_{0}$ is the residual magnetism in the machine.

To solve (4) the subdomain technique is applied. This technique consists of two steps.

The first steps divides the machine in a number of regions, called subdomains, in which the governing equation is greatly simplified. Figure 2 shows the subdomains in a slotted and in a slotless machine. They are indicated with an index $\nu$, ranging from 1 till 4 in the slotless machine and 1 till 5 in the slotted machine. Note that, in slotted machines, every slot and every slot opening is a separate subdomain. These domains are represented by indices $4 i$ and $5 i$, where $i$ refers to the slot number. Subdomain 2 is the SC. Not every machine is equipped with a shielding cylinder, however whether or not a $\mathrm{SC}$ is present in the machine has no influence in this work.

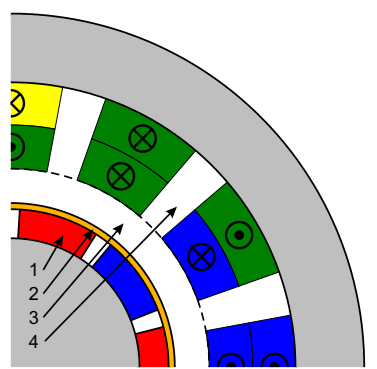

(a) Slotless machine

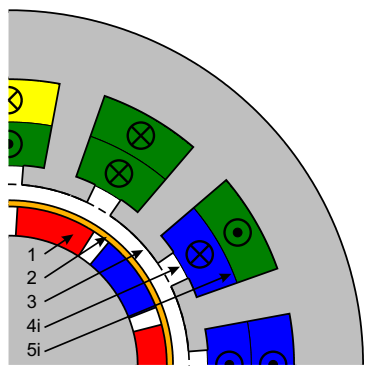

(b) Slotted machine
Fig. 2: Subdomains in slotless and slotted machines

After having obtained equations for the MVP in each of the subdomains $\left(\mathbf{A}^{(\nu)}\right)$, boundary conditions are imposed to determine the integration constants that were introduced when solving (4):

$$
\begin{array}{r}
\mathbf{n} \cdot\left(\nabla \times\left(\mathbf{A}^{(\nu)}-\mathbf{A}^{(\nu+1)}\right)\right)=0 \\
\mathbf{n} \times\left(\nabla \times\left(\frac{\mathbf{A}^{(\nu)}}{\mu^{(\nu)}}-\frac{\mathbf{A}^{(\nu+1)}}{\mu^{(\nu+1)}}\right)-\left(\frac{\mathbf{B}_{0}^{(\nu)}}{\mu^{(\nu)}}-\frac{\mathbf{B}_{0}^{(\nu+1)}}{\mu^{(\nu+1)}}\right)\right)=\mathbf{J}_{s}
\end{array}
$$

where $\nu$ and $\nu+1$ are neighboring subdomains and $\mathbf{n}$ is the unit vector normal to their boundary.

Note that the amount of integration constants that have to be determined largely determines the model's computational burden.

The solution of the simplified differential equation is obtained using the separation-of-variables technique. The equation for the magnetic vector potential is thus written as the multiplication of a $r$-, a $\phi$ - and a $t$-dependent part. The form of these parts depends on the differential equation and the spatial periodicity in the considered subdomain.

The actual form of the $r$-dependent part is not of great importance in this work. It is however important to note that the $r$-dependent part contains the integration constants, introduced when solving (4).

In Section II-A, the $\phi$-dependent part of harmonic combination $(n, k)$ was written as $e^{j k \phi}$, based on the assumption that the magnetic field has a spatial periodicity of $2 \pi$ mechanical radians. This notation holds in the subdomains that span $2 \pi$ mechanical radians. However, in slotted machines the subdomain technique introduces domains that do not span the entire $2 \pi$ mechanical radians, i.e. the slot openings and the slots. As discussed in [3], [5], [6], the magnetic fields in these subdomains have a basic spatial period of twice their respective opening angles, $2 \delta$ for the slot openings and $2 \varepsilon$ for the slots. The $\phi$-dependent part of the magnetic vector potential is then written as:

$$
\begin{cases}e^{j \frac{u \pi}{\delta}\left(\phi-\delta_{i}\right)} & \text { if } \nu=4 i \\ e^{j \frac{v \pi}{\varepsilon}\left(\phi-\varepsilon_{i}\right)} & \text { if } \nu=5 i\end{cases}
$$

To avoid confusion, a different notation for the spatialharmonic order is used; $u$ and $v$ instead of $k$. Note as well that the starting angles of the slot opening and the slot, shown in Figure 1, are subtracted from the angular coordinate. This is done to easily comply with the imposed boundary conditions [3], [5], [6].

The spatial division of the machine's geometry has no effect on the basic time period. The $t$-dependent part will always be written as $e^{-j n \omega_{m} t}$.

The above implies that the magnetic vector potential in every subdomain can be written as:

$$
A^{(\nu)}(r, \phi, t)=\sum_{n=-\infty}^{\infty} \sum_{k=-\infty}^{\infty} A_{n, k}^{(\nu)}(r) e^{j\left(\frac{k}{\left.T^{(\nu)}\left(\phi-\phi_{0}^{(\nu)}\right)-n \omega_{m} t\right)}\right.}
$$

Where $T^{(\nu)}$ is the subdomain's spatial period, i.e. $2 \delta$ in the slot openings, $2 \epsilon$ in the slots and $2 \pi$ in the other subdomains. The starting angle of subdomain $\nu$, i.e. $\delta_{i}$ in the slot openings, $\epsilon_{i}$ in the slots and 0 in the other subdomains is indicated as $\phi_{0}^{(\nu)}$.

A more extensive description of the subdomain technique is provided in [1]-[6] and is beyond the scope of this work.

Note that, although Sprangers [14] and Dubas [15] have recently introduced techniques to account for finite permeabilities, the Fourier-based model of this section assumes that the permeability of slotted structures is infinite. This assumption is more strict than the assumption of homogeneous materials posed in Section I-A, but that doesn't affect the validity of the results. Moreover, the assumption of infinite permeabilities is used in the vast majority of Fourier-based models [1]-[6].

\section{HARMONIC CONTENT IN SYNCHRONOUS ELECTRIC MACHINES}

In (7), every time- and spatial-harmonic order is considered. However, usually not every harmonic combination is present in the machine's magnetic field. Moreover, the 
harmonic content of synchronous electric machines can be predicted. An extensive discussion on this prediction is presented in [10]. This section is limited to a brief introduction on the topic so that the results can be used in Section V.

There are four aspects that define the machine's harmonic content; the permanent magnets, the stator currents, the winding distribution and the machine's geometry. These four aspects can be divided in two categories: the source terms, i.e. the magnets and the stator currents, and the spatial aspects, i.e. the machine's geometry and the winding distribution. The source terms determine which time-harmonic orders are present in the machine's magnetic field. Indeed, they can only introduce time-harmonic orders that are present in their own harmonic spectrum; $h_{m}$ for the magnets and $h_{c}$ for the stator currents. Based on the time harmonic content, the spatial aspects determine the machine's spatial harmonic content. The latter is argued by noting that the machine's magnetic field will be equal but rotated over a certain angle after a given time span. This rotational angle and time span are determined by the machine's spatial aspects.

\section{A. No-load situation}

The above can easily be explained for the no-load case of a machine with identical slots. If $N_{s}$ is the number of slots, the rotor will have rotated over one slot pitch after $\frac{T_{m}}{N_{s}}$ seconds. The magnets are then rotated over $\frac{2 \pi}{N_{s}}$ mechanical radians while the flux produced by these magnets experiences the same magnetic reluctance. This means that, from the stator point of view, the magnetic field will be identical but rotated over $\frac{2 \pi}{N_{s}}$ radians. Note that not every electric machine is equipped with $N_{s}$ equal slots. However, if the slots are unequal there is always a repetition in the shape of the slots. If there are $N_{s, e q}$ similar sets of $N_{t}$ subsequent slots, the rotational angle becomes $\frac{2 \pi}{N_{s, e q}}$ radians and the time period becomes $\frac{T_{m}}{N_{s, e q}}$ seconds. This can be expressed mathematically:

$$
A\left(r, \phi, t_{0}\right)=A\left(r, \phi+\frac{2 \pi}{N_{s, e q}}, t_{0}+\frac{T_{m}}{N_{s, e q}}\right)
$$

Moreover, the above is valid for every harmonic combination separately:

$$
\begin{aligned}
& A_{n, k}(r) e^{j\left(k \phi-n \omega_{m} t_{0}\right)}= \\
& \quad A_{n, k}(r) e^{j\left(k\left(\phi+\frac{2 \pi}{N_{s, e q}}\right)-n \omega_{m}\left(t_{0}+\frac{T_{m}}{N_{s, e q}}\right)\right)}
\end{aligned}
$$

Considering that $\omega_{m} T_{m}=2 \pi$, (9) implies a relation between the time- and spatial-harmonic orders.

$$
k-n=c N_{s, e q}
$$

where $c$ is an integer.

This means that, for the no-load situation, the time-harmonic orders are determined by the harmonic spectrum of the magnets while the machine geometry, i.e. the slots, determine the present spatial-harmonic orders. The harmonic combinations then have to satisfy (10) while $n \in h_{m}$.

\section{B. Armature-reaction situation}

For the armature-reaction field a similar approach can be made. The time-harmonic orders in the magnetic field are now determined by the harmonic spectrum of the applied currents $\left(n \in h_{c}\right)$. Let $m$ be the number of phases and $\tau$ the machine's period, i.e. the greatest common divisor of the number of pole pairs $(p)$ and the number of slots $\left(N_{s}\right)$ :

$$
\tau=\operatorname{gcd}\left(p, N_{s}\right)
$$

It was shown in [10] that, if the machine's winding is determined using the SOS, it contains $\varsigma m \tau$ slot groups (also known as phase belts). Where $\varsigma=1$ or $\varsigma=2$, depending on whether $\frac{N_{s}}{\tau}$ is odd or even. The spatial shift of a slot group corresponds to the time shift of the currents flowing through that slot group. This means that the machine's magnetic field will be equal but rotated over one slot group after $\frac{T_{m}}{m \tau}$ or $\frac{T_{m}}{2 m \tau}$ seconds. That periodicity of the armature-reaction field is mathematically expressed as:

$$
\begin{aligned}
& A_{n, k}(r) e^{j\left(k \phi-n \omega_{m} t_{0}\right)}= \\
& \quad A_{n, k}(r) e^{j\left(k\left(\phi+\frac{2 \pi}{\varsigma m \tau}\right)-n \omega_{m}\left(t_{0}+\frac{T_{m}}{\varsigma m \tau}\right)\right)}
\end{aligned}
$$

From which the following relation between the time- and spatial-harmonic orders is obtained:

$$
k-n=c \varsigma m \tau
$$

This means that under armature-reaction conditions, the harmonic combinations in the magnetic field have to satisfy (13) while $n \in h_{c}$.

\section{Load situation}

Finally, since saturation is neglected, the load field is the superposition of the no-load and armature-reaction fields. The time-harmonic orders are now introduced by both the rotor magnets and the stator currents. As mentioned, the effect of the geometry is already incorporated in the winding distribution. This means that, depending on whether $\frac{N_{s}}{\tau}$ is odd or even, the magnetic field is identical but rotated over $\frac{2 \pi}{m \tau}$ or $\frac{\pi}{m \tau}$ mechanical radians after $\frac{T_{m}}{m \tau}$ or $\frac{T_{m}}{2 m \tau}$ seconds. This implies that the spatial-harmonic orders can again be predicted using (13). An overview of the resulting harmonic content in all situations is presented in Table I.

TABLE I: Harmonic content of synchronous electric machines, with $h_{m}$ and $h_{c}$ the time-harmonic spectra of the magnets and the stator currents.

\begin{tabular}{lll}
\hline Operation & Time-HO (n) & Spatial-HO (k) \\
\hline No load & $n \in h_{m}$ & $k-n=c N_{s, e q}$ \\
Armature reaction & $n \in h_{c}$ & $k-n=c \varsigma m \tau$ \\
Load & $n \in h_{m} \cup h_{c}$ & $k-n=c \varsigma m \tau$ \\
\hline
\end{tabular}

\section{SimPlify ING THE GEOMETRY}

As already mentioned, a demand for low computational time is one of the major reasons to develop a Fourier-based model. Such models can indeed be very fast. However, their computational time is mainly determined by the amount of 
integration constants that have to be calculated. Which, in turn, depends on the amount of harmonic combinations that are taken into account. Since more accuracy implies more harmonic combinations, a strict demand for accuracy may result in unacceptable computational times. This is especially true for models that directly take into account the timedependency and for models which' purpose is an optimization procedure.

Apart from the required accuracy, the geometry has an impact on the computational time as well. Indeed, in machines with semi-closed slots, every slot opening and every slot is a separate subdomain. Each of those subdomains implies additional integration constants and therefore additional computational time. This may be a reason to simplify the geometry of machines with semi-closed slots to a similar geometry with open slots. As illustrated in Figure 3, this is done by setting the opening angle of the slots to that of the original slot openings.

Evidently, this implies that the current density has to be recalculated to keep the total current constant.

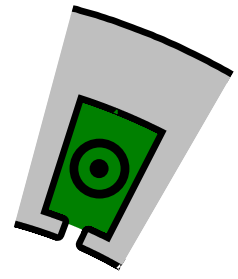

(a) Original slot

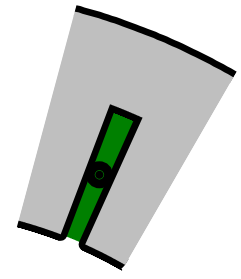

(b) Simplified slot
Fig. 3: Simplification of the machine with semi-closed slots

Section VI will verify that simplifying the slots may strongly reduce the computational time without compromising the accuracy.

\section{HARMONICS AND FOURIER-BASED MODELS}

The goal of this section is to reduce the computational time of Fourier-based models by applying the findings from Sections II and III. In a first part the harmonic combinations are reduced using Table I. In the second part the interdependency of the slots is discussed. Thirdly the real nature of the magnetic vector potential is discussed.

\section{A. Harmonic combinations}

When simulating a machine using a Fourier-based model, the number of considered time- and spatial-harmonic orders is always limited. The cut-off harmonic orders, i.e. the highest time- and spatial-harmonic orders that are taken into account, have to be carefully chosen. On the one hand, a sufficient amount of harmonic orders is required to obtain accurate results. On the other hand, the amount of considered harmonic orders should be as low as possible in order to avoid excessive computational times. However, by applying the results of Section III the amount of harmonic combinations can be reduced without affecting the accuracy. Indeed the field components related to harmonic combinations that do not satisfy the requirements of Table I will be zero. These combinations can thus be disregarded, doing so results in a first harmonicrelated reduction of the computational burden.

Note that the imposed relations between the time- and spatialharmonic combinations were found assuming a basic spatial period of $2 \pi$ mechanical radians. Therefore Table $\mathrm{I}$ is not valid in the slot openings and the slots of a slotted machine.

\section{B. Interdependence of the slots}

Although Table I does not apply to the magnetic vector potential in the slots and the slot openings, the time periodicities found in Section III are still valid for the machine's total magnetic field.

Equation (8), for example, implies that under no-load conditions the magnetic field in slot opening $i+N_{t}$ lags the magnetic field in slot opening $i$ by $\frac{T_{m}}{N_{s, e q}}$ seconds. A relation can then be found between the magnetic vector potential in these slot openings:

$$
A^{(4 i)}\left(r, \phi, t_{0}\right)=A^{\left(4 i+N_{t}\right)}\left(r, \phi+\frac{2 \pi}{N_{s, e q}}, t_{0}+\frac{T_{m}}{N_{s, e q}}\right)
$$

Which is again valid for every harmonic combination separately:

$$
\begin{aligned}
& A_{n, u}^{(4 i)}(r) e^{j\left(\frac{u \pi}{\varepsilon}\left(\phi-\varepsilon_{i}\right)-n \omega_{m} t_{0}\right)}= \\
& \quad A_{n, u}^{\left(4 i+N_{t}\right)}(r) e^{j\left(\frac{u \pi}{\varepsilon}\left(\phi+\frac{2 \pi}{N_{s}, e q}-\varepsilon_{i+N_{t}}\right)-n \omega_{m}\left(t_{0}+\frac{T_{m}}{N_{s}, e q}\right)\right)}
\end{aligned}
$$

and since $\varepsilon_{i+N_{t}}=\varepsilon_{i}+\frac{2 \pi}{N_{s, e q}}$ :

$$
A_{n, u}^{(4 i)}(r)=A_{n, u}^{\left(4 i+N_{t}\right)}(r) e^{-j n \frac{2 \pi}{N_{s, e q}}}
$$

A similar relation can be found between the Fourier coefficients of the slots:

$$
A_{n, v}^{(5 i)}(r)=A_{n, v}^{\left(5 i+N_{t}\right)}(r) e^{-j n \frac{2 \pi}{N_{s, e q}}}
$$

For the armature-reaction and load fields, a similar periodicity was found. Analogously as in the above, the following relations can be found:

$$
A_{n, u}^{(4 i)}(r)=A_{n, u}^{\left(4 i+\frac{N_{s}}{\varsigma m \tau}\right)}(r) e^{-j n \frac{2 \pi}{\varsigma m \tau}}
$$

and

$$
A_{n, v}^{(5 i)}(r)=A_{n, v}^{\left(5 i+\frac{N_{s}}{\varsigma m \tau}\right)}(r) e^{-j n \frac{2 \pi}{\varsigma m \tau}}
$$

Equations (16)-(19) show a relation between the Fourier coefficients of different slot openings and slots. This implies that fewer coefficients have to be calculated using the boundary conditions. A second harmonic-related technique to reduce the computational time is thus to account for the relation between integration constants of different slots. This lower computational time is again achieved without loss of accuracy.

\section{Real functions}

Although (7) contains complex components, the magnetic vector potential as such is real. This implies that the Fourier coefficients linked to harmonic combinations $(n, k)$ 
and $(-n,-k)$ have to be complex conjugate. For the magnetic vector potential this implies:

$$
A_{n, k}^{(\nu)}(r)=\left(A_{-n,-k}^{(\nu)}(r)\right)^{*}
$$

Note that this is valid for every subdomain.

The above relation implies that only half of the integration constants have to be calculated, which results in a large reduction of the computational time.

\section{VALIDATION AND BENCHMARKING}

In addition to the computational-time reduction techniques described in the above, some authors already account for the machine's periodicity $(\tau)$ in order to reduce the computational burden. This implies that, in subdomains that span the entire $2 \pi$ mechanical radians, they only consider time- and spatialharmonic orders that are multiples of $\tau$. Moreover, only $\frac{N_{s}}{\tau}$ slots and slot openings have to be taken into account. This approach is, in effect, a simple version of the preliminary harmonic analysis of Section V. To evaluate the effect of accounting for periodicity and the techniques proposed in Sections IV and V, several example machines are simulated using four different models:

- original model: the model presented in [5]

- slots model: the original model with simplified slots

- periodic model: the model that accounts for the machine's periodicity

- harmonics model: the model that accounts for the preliminary harmonic study

It was already mentioned that, for the sake of generality, this work considers Fourier-based models with a direct timedependency. However, the vast majority of Fourier-based models in literature still uses time stepping. Therefore, this section also considers an application of the above for the case of time-stepped FB models.

\section{A. Direct time-dependency}

The first example machine is a three-phase inner-rotor PMSM with distributed windings. The second example machine has an outer-rotor topology and five phases that are arranged in a fractional-slot concentrated winding. A cross section of both machines is shown in Figure 4. Their parameters are listed in Table II, where RY indicates the rotor yoke, PM the permanent magnets, $\mathrm{SC}$ the shielding cylinder, AG the air gap, SO the slot openings, SL the slots and SY the stator yoke. Note that, depending on whether no-load or armature-reaction conditions are regarded, $B_{0}$ is 1.2 or $0 \mathrm{~T}$ and $J$ is 0 or $5 \frac{A}{\mathrm{~mm}^{2}}$. The magnets are radially magnetized and the applied current is sinusoidal.

The highest time-harmonic order is set to 130. In the circular subdomains $(\nu=1 \cdots 3)$, the highest spatial-harmonic order is 130 as well. In the slots and the slot openings, the highest spatial harmonic order is set to 15 . The induction in the air gap can be calculated using the definition of the magnetic vector potential:

$$
\mathbf{B}=\nabla \times \mathbf{A}
$$

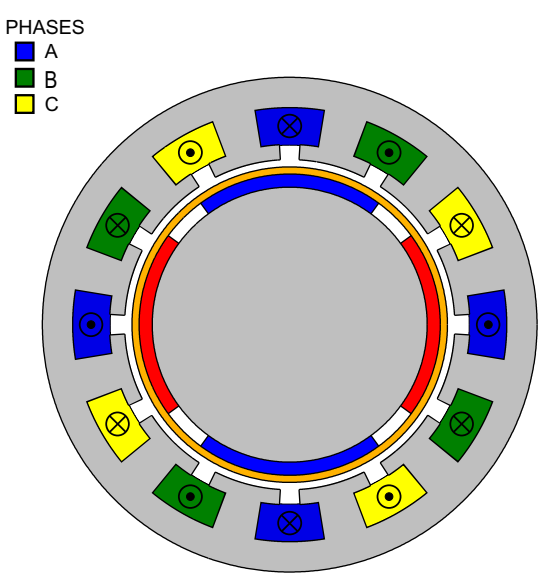

(a) Inner-rotor machine

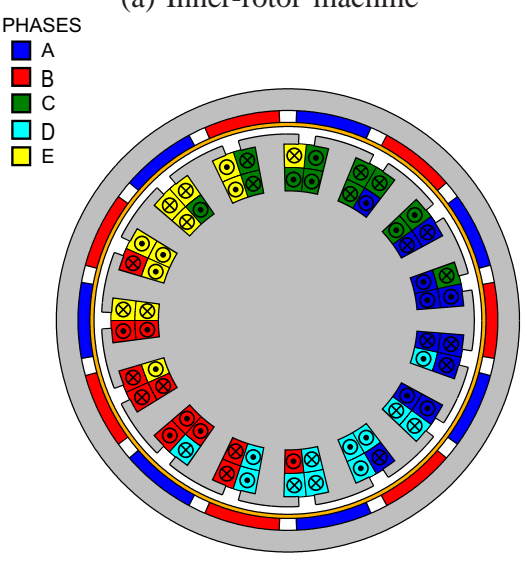

(b) Outer-rotor machine

Fig. 4: Studied machines

TABLE II: Parameters of the two PMSM machines

\begin{tabular}{lrr}
\hline Parameter & Inner rotor & Outer rotor \\
\hline Number of slots $\left(N_{s}\right)$ & 12 & 15 \\
Number of pole pairs $(p)$ & 2 & 7 \\
Number of phases $(m)$ & 3 & 5 \\
Angular span of a magnet $\left(\phi_{m}\right)$ & $72.00^{\circ}$ & $20.57^{\circ}$ \\
Radius RY - external $\left(r_{0}\right)$ & $0.00 \mathrm{~mm}$ & $79.20 \mathrm{~mm}$ \\
Radius RY - PM $\left(r_{1}\right)$ & $47.25 \mathrm{~mm}$ & $72.20 \mathrm{~mm}$ \\
Radius PM - SC $\left(r_{2}\right)$ & $52.25 \mathrm{~mm}$ & $67.70 \mathrm{~mm}$ \\
Radius SC - AG $\left(r_{3}\right)$ & $54.25 \mathrm{~mm}$ & $66.70 \mathrm{~mm}$ \\
Radius AG - SO $\left(r_{4}\right)$ & $57.25 \mathrm{~mm}$ & $63.70 \mathrm{~mm}$ \\
Radius SO - SL $\left(r_{5}\right)$ & $62.00 \mathrm{~mm}$ & $60.70 \mathrm{~mm}$ \\
Radius SL - SY $\left(r_{6}\right)$ & $75.20 \mathrm{~mm}$ & $44.20 \mathrm{~mm}$ \\
Radius SY external $\left(r_{7}\right)$ & $85.20 \mathrm{~mm}$ & $0.00 \mathrm{~mm}$ \\
Opening angle of the slot openings $(\delta)$ & $6.93^{\circ}$ & $5.54^{\circ}$ \\
Opening angle of the slots $(\epsilon)$ & $18.78^{\circ}$ & $15.02^{\circ}$ \\
Residual magnetism $\left(B_{0}\right)$ & 1.2 or $0 \mathrm{~T}$ \\
Current density in the slots $(\mathrm{RMS})(J)$ & 0 or $5 \frac{A}{\mathrm{~mm} m^{2}}$ \\
Frequency of the applied current $(f)$ & $1000 \mathrm{~Hz}$ \\
Conductivity of the SC $\left(\sigma_{S C}\right)$ & $5.96 .10^{7} \frac{S}{m}$ \\
\hline
\end{tabular}

Table III shows the computational times of the performed simulations for both the no-load (NL) and the armature-reaction (AR) conditions. All of the calculations were performed on a quad core PC with a clock rate of $2.83 \mathrm{GHz}$ and a memory of $8 \mathrm{~GB}$. To evaluate the accuracy of the obtained solutions, they are compared with results from a $2 \mathrm{D}$ finite-element model. The \% RMS deviation, with respect to the FE model, of the 
magnetic flux density in the center of the air gap is also shown in Table III. This deviation is calculated as:

$$
d=\frac{\int_{0}^{2 \pi}\left|B_{F E}\left(r_{a c}, \phi, 0\right)-B_{F B}\left(r_{a c}, \phi, 0\right)\right| \mathrm{d} \phi}{\int_{0}^{2 \pi}\left|B_{F E}\left(r_{a c}, \phi, 0\right)\right| \mathrm{d} \phi} \cdot 100 \%
$$

Note that the computational time of the FE models strongly depends on the modeled geometry, the number of time steps required for the calculations to converge and even the applied software package. In this work, computational times ranging between 4 seconds, for the magnetostatic calculations of Section VI-B, and over 2500 seconds, for some of the magnetoquasistatic calculations of Section VI-A, were required. However, as the focus is on FB models, the computational time of the FE models will not be discussed in detail.

It can readily be seen from Table III that there is a significant difference in required computational time between the original model and the model with simplified slots. Nevertheless, the simplified model can predict the flux density in the center of the air gap with an accuracy that is comparable to that of the original model. However, it is evident that the effect on the in the slots will not be negligible. Simplifying the geometry is thus an interesting technique for FB models that study quantities such as torque and magnet losses, i.e. quantities that are not strongly affected by the field in the slots. One example of a machine parameter that cannot effectively be calculated with simplified slots is the inductance.

For the inner-rotor machine, accounting for the periodicity has an even greater effect on the computational time. This computational-time reduction is achieved without affecting the model's accuracy. This was indeed expected; the optimized model only avoids unnecessary calculations. This doesn't affect the actual result. As the periodicity of the outerrotor machine is 1, its computational time is not affected.

The computational gain for the model that applies the preliminary harmonic analysis of Section V is by far the largest. It can also be seen from Table III that the optimized model has a significantly lower computational time for the armaturereaction field than for the field at no-load conditions. The reason is that a sinusoidal current is imposed, this implies

TABLE III: Optimized model; RMS deviation and computational time for both no-load (NL) and armature-reaction (AR) conditions

\begin{tabular}{lcccccr}
\hline Model & \multicolumn{3}{c}{ RMS deviation (\%) } & \multicolumn{2}{c}{ Computational time (s) } \\
& \multicolumn{2}{c}{$\mathrm{NL}$} & \multicolumn{2}{c}{$\mathrm{AR}$} & $\mathrm{NL}$ & $\mathrm{AR}$ \\
& $B_{r}$ & $B_{\phi}$ & $B_{r}$ & $B_{\phi}$ & & \\
\hline \multicolumn{2}{l}{ Inner-rotor machine } & & & & & \\
\hline Original & 0.042 & 2.749 & 0.035 & 0.565 & 515.71 & 517.31 \\
Slots & 0.045 & 2.734 & 0.033 & 0.571 & 335.09 & 335.32 \\
Periodic & 0.042 & 2.749 & 0.035 & 0.565 & 44.31 & 44.97 \\
Harmonics & 0.042 & 2.749 & 0.035 & 0.565 & 3.27 & 0.11 \\
\hline \multicolumn{2}{l}{ Outer-rotor machine } & & & & & \\
\hline Original & 0.025 & 0.086 & 0.111 & 0.039 & 635.59 & 636.02 \\
Slots & 0.025 & 0.091 & 0.116 & 0.037 & 439.12 & 438.78 \\
Periodic & 0.025 & 0.086 & 0.111 & 0.039 & 635.73 & 635.81 \\
Harmonics & 0.025 & 0.086 & 0.111 & 0.039 & 1.34 & 0.25 \\
\hline
\end{tabular}

that $h_{c}$ only contains the fundamental time-harmonic order. In contrast, $h_{m}$ contains much more time-harmonic orders.

Note that in slotless machines, all calculations can be performed separately for every time- and spatial-harmonic combination. This implies that more, but smaller, boundarycondition matrices are to be considered. Equations (10) and (13) will still allow to further reduce the size of the matrices. However, as the computational time depends on the square of the matrix size, this reduction will only result in a limited computational-time reduction.

In addition to the comparison of Table III, Figure 5 shows the computational times of the original model, the slots model, the harmonics model and a model that combines simplified slots with a harmonic analysis for different cut-off harmonic orders. The machine under consideration is the inner-rotor machine of Figure 4a. Note that the spatial cut-off harmonic orders in the slots are one tenth of the cut-off harmonic orders in the circular subdomains. This is common for Fourier-based models, indeed the slot opening is much smaller than $2 \pi$ radians. Figure 5 clearly shows that the preliminary harmonic study, by far, has the largest effect on the computational time. Figure 5 also shows the \% RMS deviation of the original model as a function of the amount of harmonic orders. This figure shows that a fairly good accuracy is obtained, even at a low cut-off harmonic order. Note that the RMS deviations of the other models are not shown because they are very close to that of the original model.

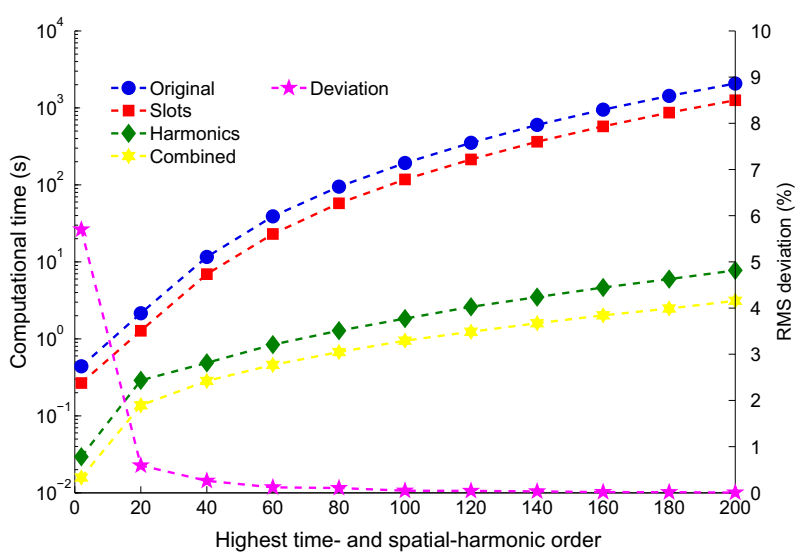

Fig. 5: Comparison of the computational times and RMS deviation at different cut-off harmonic orders

\section{B. Time stepping}

Consider the first example machine, as described by Table II and Figure 4a. However, now the shielding cylinder is replaced by air, the number of slots is set to 96 and the number of pole pairs is increased to 16 , while maintaining all geometrical proportions.

The large number of slots allows to evaluate the effect that the number of slots has on the computational time. On the other hand, the absence of a shielding cylinder allows for a time-stepped magnetostatic calculation. Which is, to date, the most commonly used Fourier-based modeling technique. Such a time-stepped magnetostatic calculation was performed 
for a single time step using all of the above four models, the results are presented in Table IV.

TABLE IV: Optimized model; RMS deviation and computational time for both no-load (NL) and armature-reaction (AR) conditions

\begin{tabular}{|c|c|c|c|c|c|c|}
\hline \multirow[t]{3}{*}{ Model } & \multicolumn{4}{|c|}{ RMS deviation (\%) } & \multicolumn{2}{|c|}{ Computational time (s) } \\
\hline & \multicolumn{2}{|c|}{ NL } & \multicolumn{2}{|c|}{$\mathrm{AR}$} & \multirow{2}{*}{ NL } & \multirow{2}{*}{$\mathrm{AR}$} \\
\hline & $B_{r}$ & $B_{\phi}$ & $B_{r}$ & $B_{\phi}$ & & \\
\hline \multicolumn{7}{|c|}{ Inner-rotor machine with 16 pole pairs } \\
\hline Original & 0.087 & 0.364 & 0.430 & 0.184 & 173.61 & 159.77 \\
\hline Slots & 0.091 & 0.361 & 0.427 & 0.183 & 10.23 & 10.19 \\
\hline Periodic & 0.087 & 0.364 & 0.430 & 0.184 & 1.70 & 1.49 \\
\hline Harmonics & 0.087 & 0.364 & 0.430 & 0.184 & 0.72 & 0.60 \\
\hline
\end{tabular}

First and foremost, it should be noted that the computational times of Table IV are not simply comparable with those of Table III. Whereas the calculations from the previous section cover a complete time period of the machine, the calculations presented here only cover a single time step.

Nevertheless, some interesting conclusions can be drawn. Firstly, it can be seen that the technique of simplifying the slots now results in a much larger reduction of the computational time. The reason is that, due to their larger number, the effect of the slots on the computational time is much larger. Next, it can be seen that, under armature-reaction conditions, the computational time of the harmonic model has the same order of magnitude as that of the harmonic model with direct time-dependency. This could indeed have been expected; as the current is sinusoidal, the time-dependent harmonic model only has to consider a single harmonic order. This implies that, like for the time-stepped model, only a single system of boundary conditions has to be solved.

\section{CONCLUSIONS}

This work started with an introduction on Fourier-based modeling in Section II and the basics on harmonic content of synchronous electric machines in Section III.

Using the information from these two sections and some general considerations on the magnetic field, two major techniques to reduce the computational burden of FB model were proposed; simplifying the geometry and accounting for a preliminary harmonic analysis.

Simplifying the geometry proved to result in a significant reduction of the computational time while barely affecting the accuracy of the flux density in the center of the air gap. In Section V, the harmonic analysis of [10] was used to propose three harmonic-related techniques to reduce the computational time. A first harmonic-related technique is to only consider the harmonic combinations that are actually present in the magnetic field. Secondly a relation between the magnetic field in different slots can be found. This relation reduces the amount of integration constant that have to be calculated. Finally, it was argued that the auxiliary quantities that describe the magnetic field have to be real. This also imposes a relation between different integration constants. The above described techniques were successfully validated using a FEM. Finally, a benchmark test was done to compare the computational time of the standard Fourier-based model with the optimized models. A huge reduction in computational time was noted. Although the focus was on models that directly take into account the time-dependency, an additional example for time-stepped models was included. Moreover, studying the presence of harmonic combinations also results in a better insight in the machine's operation.

The presented work thereby contributes to the development of a faster simulation tool and to the understanding of synchronous machines.

\section{REFERENCES}

[1] Z. Zhu, L. J. Wu, and Z. Xia, "An accurate subdomain model for magnetic field computation in slotted surface-mounted permanentmagnet machines," IEEE Transactions on Magnetics, vol. 46, no. 4, pp. 1100-1115, Apr. 2010.

[2] Z. Zhu, D. Howe, and C. Chan, "Improved analytical model for predicting the magnetic field distribution in brushless permanent-magnet machines," IEEE Transactions on Magnetics, vol. 38, no. 1, pp. 229238, Jan. 2002

[3] B. L. J. Gysen, K. Meessen, J. J. H. Paulides, and E. Lomonova, "General formulation of the electromagnetic field distribution in machines and devices using fourier analysis," IEEE Transactions on Magnetics, vol. 46, no. 1, pp. 39-52, Jan. 2010.

[4] S. R. Holm, H. Polinder, and J. Ferreira, "Analytical modeling of a permanent-magnet synchronous machine in a flywheel," IEEE Transactions on Magnetics, vol. 43, no. 5, pp. 1955-1967, May 2007.

[5] B. Hannon, P. Sergeant, and L. Dupré, " $2 d$ analytical subdomain model of a slotted PMSM with shielding cylinder," IEEE Transactions on Magnetics, vol. 50, no. 7, pp. 1-11, 2014.

[6] T. Lubin, S. Mezani, and A. Rezzoug, "2-d exact analytical model for surface-mounted permanent-magnet motors with semi-closed slots," IEEE Transactions on Magnetics, vol. 47, no. 2, pp. 479-492, Feb. 2011.

[7] A. Bellara, H. Bali, R. Belfkira, Y. Amara, and G. Barakat, "Analytical Prediction of Open-Circuit Eddy-Current Loss in Series Double Excitation Synchronous Machines," IEEE Transactions on Magnetics, vol. 47, no. 9, pp. 2261-2268, Sep. 2011.

[8] H. Tiegna, A. Bellara, Y. Amara, and G. Barakat, "Analytical Modeling of the Open-Circuit Magnetic Field in Axial Flux Permanent-Magnet Machines With Semi-Closed Slots," IEEE Transactions on Magnetics, vol. 48, no. 3, pp. 1212-1226, Mar. 2012.

[9] K. Ramakrishnan, D. Zarko, A. Hanic, and G. Mastinu, "An Improved Method for Field Analysis of Surface Permanent MagnetMachines Using Schwarz-Christoffel Transformation," IET Electric Power Applications, Feb. 2017. [Online]. Available: http://digital-library. theiet.org/content/journals/10.1049/iet-epa.2016.0712

[10] B. Hannon, P. Sergeant, and L. Dupré, "Time- and Spatial-Harmonic Content in Synchronous Electrical Machines," IEEE Transactions on Magnetics, vol. PP, no. 99, pp. 1-1, 2016.

[11] P. Arumugam, F. Dubas, and C. Gerada, "Estimation of eddy current loss in semi-closed slot vertical conductor permanent magnet synchronous machines considering eddy current reaction effect," IEEE Trans. Magn., vol. 49, no. 10, pp. 5326-5335, Oct. 2013.

[12] P.-D. Pfister and Y. Perriard, "Slotless Permanent-Magnet Machines: General Analytical Magnetic Field Calculation," IEEE Transactions on Magnetics, vol. 47, no. 6, pp. 1739-1752, Jun. 2011.

[13] P.-D. Pfister, X. Yin, and Y. Fang, "Slotted Permanent-Magnet Machines: General Analytical Model of Magnetic Fields, Torque, Eddy Currents and Permanent Magnet Power Losses including the Diffusion Effect," IEEE Transactions on Magnetics, vol. 52, no. 5, pp. 1-13, 2016.

[14] R. Sprangers, J. Paulides, B. Gysen, and E. Lomonova, "Towards Magnetic Saturation in Semi-Analytical Harmonic Modeling for Electric Machine Analysis," IEEE Transactions on Magnetics, vol. 2, no. 52, pp. 1-10, 2016.

[15] F. Dubas and K. Boughrara, "New Scientific Contribution on the 2-D Subdomain Technique in Cartesian Coordinates: Taking into Account of Iron Parts," Mathematical and Computational Applications, vol. 22, no. 1, p. 17, Feb. 2017. [Online]. Available: http://www.mdpi.com/2297-8747/22/1/17 


\section{BIOGRAPHIES}

Bert Hannon received the M.Sc. degree in electrotechnical engineering in 2011 from the University College Ghent, Ghent, Belgium. In 2012, he started a Ph.D. study at the Electrical Energy Laboratory of Ghent University. His research interests lies in the analytical modeling of electric machines, with a focus on high-speed machines.

Peter Sergeant received the M.Sc. degree in electromechanical engineering in 2001, and the Ph.D. degree in engineering sciences in 2006, both from Ghent University, Ghent, Belgium. In 2001, he became a researcher at the Electrical Energy Laboratory of Ghent University. He became a postdoctoral researcher at Ghent University in 2006 and at Ghent University College in 2008. Since 2012, he is associate professor at Ghent University. His current research interests include numerical methods in combination with optimization techniques to design nonlinear electromagnetic systems, in particular, electric machines for sustainable energy applications.

Luc Dupré received the electrical and mechanical engineering degree and the Ph.D. degree in applied sciences from the Ghent University, Belgium, in 1989 and 1995, respectively. In 1989, he joined the Department of Electrical Energy, Systems and Automation, Ghent University, as a research assistant. In 1996, he was a postdoctoral researcher for the Fund of Scientific Research-Flanders and in 1998 for six months a visiting post-doctoral fellow at Istituto Elettrotecnico Nazionale 'Galileo Ferraris', in Torino (Italy). In 2002, he became an associate professor at the Faculty of Engineering and Architecture, Ghent University and since 2006 he is full professor at that faculty. His research interests mainly include numerical methods for low frequency electromagnetism (in particular for electric machines), modeling and characterization of soft magnetic materials, micromagnetism, inverse problems and optimization in electromagnetism. 